\title{
Patients' subjective initial response and the outcome of inpatient and day hospital treatment
}

\section{Authors:}

\section{1) Stefan Priebe, Prof. Dr. AUTHOR FOR CORRESPONDENCE}

Affiliation: Queen Mary University of London, Barts' and the London School of Medicine, Unit for Social and Community Psychiatry, London, United Kingdom.

Address: Academic Unit, Cherry Tree Lane, Glen Road, London E13 8SP, United Kingdom. Email: s.priebe@qmul.ac.uk

Telephone: $0044-20-7540-6755$

Fax: $0044-20-7540-2976$

\section{2) Kirsten Barnicot, B.A.}

Affiliation: Queen Mary University of London, Barts' and the London School of Medicine, Unit for Social and Community Psychiatry, London, United Kingdom.

Address: Academic Unit, Cherry Tree Lane, Glen Road, London E13 8SP, United Kingdom Email: k.barnicot@qmul.ac.uk

Telephone: $0044-20-7540-6755$

Fax: $0044-20-7540-2976$

\section{3) Rose McCabe, Ph.D}

Affiliation: Queen Mary University of London, Barts' and the London School of Medicine, Unit for Social and Community Psychiatry, London, United Kingdom.

Address: Academic Unit, Cherry Tree Lane, Glen Road, London E13 8SP, United Kingdom Email: r.mccabe@qmul.ac.uk

Telephone: $0044-20-7540-6755$

Fax: $0044-20-7540-2976$

\section{4) Andrej Kiejna, Prof. Dr.}

Affiliation: Department of Psychiatry, Wroclaw Medical University

Address: Department of Psychiatry, Wroclaw Medical University, L. Pasteura str. 10, 50-367

Wroclaw, Poland

Email: akiejna@psych.am.wroc.pl

Telephone: 0048-71-784-1600

Fax: 0048-71-784-1602

5) Petr Nawka, M.U.Dr.

Affiliation: Michalovce Psychiatric Hospital, Michalovce, Slovak Republic 
Address: Hopfgartenstr.16, D-01307 Dresden

Email: nawka@pnmi.sk

Telephone: 0049-351-459-0072

Fax: 0049-351-440-3129

6) Jiri Raboch, Prof. Dr.

Affiliation: Department of Psychiatry, Charles University in Prague.

Address: Head, Department of Psychiatry, Charles University in Prague, $1^{\text {st }}$. Faculty of Medicine and General Teaching Hospital, Ke Karlovu 11, 12000 Praha 2, Czech Republic. Email: raboch.jiri@vfn.cz

Telephone: 0042-022-491-6858

Fax: 0042-022-492-3077

7) Matthias Schützwohl, P.h.D.

Affiliation: Technishche Universitat Dresden, Department of Psychiatry and Psychotherapy Address: Technische Universität Dresden, Department of Psychiatry and Psychotherapy, Fetscherstraße 74 D - 01307 Dresden, Germany

Email: Matthias.Schuetzwohl@uniklinikum-dresden.de

Telephone: 0049 -351- 458-5490

Fax: $0049-351-449-210-276$

8) Thomas Kallert, Prof., Dr.

Affiliation: Department of Psychiatry, Psychosomatic Medicine and Psychotherapy, Park Hospital Leipzig.

Address: Head of the Department of Psychiatry, Psychosomatic Medicine and Psychotherapy, Park Hospital Leipzig, Morawitzstraße 2, D-04289 Leipzig, Germany

Email: thomas.kallert@parkkrankenhaus-leipzig.de

Telephone: 0049-341-864-1260

Fax: 0049-341-864-1265 
Objective: This study aimed to establish whether psychiatric patients' subjective initial response (SIR) to hospital and day hospital treatment predicts outcomes over a one year follow-up period.

Method: We analysed data from 765 patients who were randomised to acute psychiatric treatment in a hospital or day hospital. SIR was assessed on day three after admission. Outcomes were psychiatric symptom levels and social disability at discharge, and at 3 and 12 months after discharge.

Results: After controlling for socio-demographic and clinical characteristics, a more positive SIR was significantly associated with lower symptom levels at discharge and 3 months after discharge, and lower social disability at 3 months and 12 months after discharge.

Conclusion: SIR can predict outcomes of complex interventions over a one year period. Patients' initial views of acute hospital and day treatment should be elicited and considered as important. 


\section{Introduction}

The concept of subjective initial response (SIR), defined as patients' initial assessment of the appropriateness and effects of their treatment, originated from psychopharmacology studies conducted in the 1970s. Van Putten and May found that the SIR of patients with schizophrenia to neuroleptic medication was significantly associated with clinical outcome at the end of a four week treatment period (27). Patients who stated within two days of a new treatment that they believed their medication to be right for them and helpful had lower symptom levels at the end of treatment, a finding that was repeatedly replicated $(1,2,25,26)$.

The idea that patients' SIR may predict outcome was subsequently extended to complex interventions. A first publication showed that a more positive SIR of depressed patients to psychiatric hospital treatment was correlated with lower symptom levels four months after admission (14). Further studies suggested that SIR may also predict the outcome of hospital and day hospital treatment in patients with schizophrenia $(3,17)$. The predictive value of SIR was independent of patients' initial symptom improvement $(17,18)$ and their psychiatrist's expectations regarding the likely success of treatment (18).

All these studies assessed patients' SIR within a maximum of three days after admission and found a significant association of more positive SIR with more positive outcomes. However, this research has serious limitations. All studies were single site studies in the same country (Germany) and had relatively small sample sizes (up to a maximum of $n=63(18)$ ). Only one study assessed outcome beyond discharge, using a four month follow-up period (14). Patient characteristics as potentially confounding variables were not controlled for in any study. Thus, there is not yet sufficient 
evidence for a generalised conclusion that asking patients for their views of treatment shortly after admission really provides information that is predictive of outcomes.

The European Day Hospital Evaluation (EDEN) study, a randomised controlled trial of acute inpatient versus day hospital treatment conducted across five European countries (11), presented an ideal opportunity to improve on existing research by assessing the association between SIR to hospital or day hospital treatment and outcome in a large multi-national sample including three major diagnostic groups.

\section{Objectives}

The present study aimed to assess the association of SIR to acute hospital or day hospital treatment with levels of symptoms and social disability at discharge, and at 3 months and 12 months after discharge. We hypothesised that a more positive SIR would predict more favourable outcomes independently of socio-demographic and clinical characteristics of the patients, and that the predictive association would not differ between hospital and day hospital treatment or across major diagnostic groups.

\section{Subjects and methods}

\section{Study Design}

The present study used data from the EDEN study, a multi-centre randomised controlled trial comparing acute treatment in day hospitals with that on conventional inpatient wards in five European countries (Prague, Czech Republic; Dresden, Germany; Wroclaw, Poland; Michalovce, Slovakia; London, United Kingdom). The EDEN study found more favourable outcomes for social disability in day hospitals, and no difference in terms of symptoms or subjective quality of life (11). Here, we analysed the association between SIR and outcomes at discharge, 3 months postdischarge, and 12 months post-discharge. 


\section{Sample}

All patients aged between 18 and 65 years who were in need of acute treatment in a psychiatric facility in one of the five centres during the study period were eligible to participate in the EDEN study. Exclusion criteria were an admission only for diagnostic purposes; involuntary admission; likely requirement of coercive measures or consistent one-to-one observation; acute intoxication; a somatic disorder requiring inpatient care; direct referral from another hospital; homelessness; one-way journey to the day hospital of more than 60 minutes or requirement to be consistently accompanied on the way to and from the day hospital; incapacity to give informed consent or not giving informed consent. Study participants were randomised to acute treatment either in a day hospital or on a conventional inpatient ward. After complete description of the study to the potential participants, written informed consent was obtained. The study was approved by the relevant ethics committees. Details of the rationale, settings, methods and main findings of the study have been published elsewhere (11)

Of the 1117 patients participating in EDEN (10), those with unclear or infrequent diagnoses were excluded so that we could analyse three major psychiatric diagnostic categories (ICD-10 F20-29, F30-39 and F40-59) as potentially influential factors, leaving a total of 765 patients included in the present analysis. In line with clinical practice at participating centres, clinical diagnoses were made according to ICD-10.

\section{Outcome Measures}

For this analysis we used the primary observer-rated outcome criteria of the EDEN study, i.e. psychiatric symptom levels and social disability at discharge, and at 3 and 12 months after discharge. Self-reported outcome criteria were not included in this 
analysis in order to avoid the potentially confounding covariance between selfreported predictors and self-reported outcomes $(5,6,19)$.

Psychiatric symptom levels were assessed with the 24-item version (4.0) of the Brief Psychiatric Rating Scale (BPRS) (28). The inter-rater reliability achieved in the training of researchers in the study was 0.78 (intra-class correlation, ICC). Social disability was rated on the Groningen Social Disability Schedule, Second Revision (GSDS) (29). On the GSDS disabilities are rated in 8 social roles, with disability in each role ranging from 0 ("no disability") to 3 ("severe disability"), and a sum score is then computed. The inter-rater reliability for the sum score in this study was 0.77 (ICC) (24).

\section{Subjective Initial Response}

SIR was assessed on day 3 of admission, i.e. about two days after admission, since the day of admission counts as day 1. It was measured on the Client Assessment of Treatment Scale (CAT) (16). On the CAT, patients' rate their satisfaction with and appropriateness of treatment using a scale from 0 ("not at all") to 10 "yes, entirely", on seven dimensions: "Do you believe you are receiving the right treatment/care for you here?", "Does your psychiatrist understand you and is he/she engaged in your treatment/care?", "Are relations with other staff members here pleasant for you?", "Do you believe you are receiving the right medication for you?", "Do you believe the other elements of treatment/ care here are right for you?", "Do you feel respected and regarded well here?", and "Has treatment/care here been helpful for you?". The scale has been found to have an internal consistency of 0.90 in a previous large study in psychiatric inpatients (20). The mean score was used as the measure of SIR. 


\section{Other potential predictors}

Other potential predictors assessed were gender, living status (living alone versus with someone), employment status, age, education level, and the main psychiatric diagnosis according to ICD-10 (9). The variables were selected based on previous studies on SIR and clinical outcomes in similar patient groups $(7,8,13)$. Sociodemographic variables were assessed using the Client Socio-demographic and Clinical History Schedule (12). As a complete standardized diagnostic interview with all patients was impracticable in the case of acute admissions, we used the clinical discharge diagnosis in all cases. The main diagnoses were analysed in three categories, a) schizophrenia, schizotypal and delusional disorders (ICD: F20-29); b) mood disorders (F30-39); and c) neurotic, stress related and somatoform disorders, and behavioural syndromes associated with physiological disturbances and physical factors (F40-59).

\section{Procedure}

All predictor and outcome variables were assessed by researchers who had no involvement in treatment. Potential predictor variables other than the clinical diagnosis and SIR were assessed before randomly assigning participants to either inpatient or day hospital treatment. SIR was assessed on day 3 of admission. Outcome measures were assessed at admission, at discharge, and at 3 month and 12 month follow ups after discharge.

\section{Statistical Method}

Mixed model analyses were conducted separately at discharge, 3 months and 12 months to determine over which period of time SIR may predict outcomes. The two outcome variables, BPRS and GSDS, were analysed in separate models. Patient characteristics were included in the mixed model analyses as main effects so that the predictive association of SIR and outcomes was adjusted for the influence of those 
variables. The baseline measurement of BPRS and GSDS respectively, the treatment setting (hospital versus day hospital), the length of stay in the hospital or day hospital and the study centre (i.e. the national site of data collection in the EDEN trial) were controlled for in all multivariate analyses as potential confounders. All of these factors and the SIR were introduced as fixed effects whereas the patient was treated as a random effect. In a second step, potential interaction effects of the treatment setting (i.e. hospital versus day hospital) and diagnostic category with SIR in predicting outcomes were tested to assess whether the predictive association of SIR significantly differed between the two settings or across the three diagnostic groups.

During the trial, some patients dropped out, resulting in incomplete observations. These incomplete observations were not computed but assumed to be missing at random in the mixed effect model analysis. Additionally, the SIR of participants who dropped out was compared to the SIR of those who did not, to explore whether they differed.

All statistical analyses were carried out using Statistical Analysis System (SAS) version $9.1(23)$

\section{Results}

\section{Sample characteristics}

The characteristics of the sample are summarised in Table 1.

[Insert Table 1 about here] 
Of the total sample of 765 patients, 374 were admitted to conventional wards, and 391 to day hospitals. The majority of patients were female, and each of the three diagnostic groups (ICD-10 F20-F29, F30-F39, and F40-F59) represented more than a quarter of the sample. The mean score on the CAT on day three was 7.6.

Of all patients recruited at baseline, $87 \%$ were followed up at discharge, $78 \%$ after 3 months, and $69 \%$ after 12 months (4)

\section{Clinical Outcomes}

Table 2 presents levels of the outcome measures at four time points: baseline, discharge, three months after discharge, and twelve months after discharge.

[Insert Table 2 about here]

Symptom levels substantially reduced between baseline and discharge. Levels fluctuated between discharge and 12 months follow-up, but were at all post baseline measurements significantly less severe than at baseline. The score on the GSDS at baseline was 9.37 , and significantly improved over time.

\section{Association of SIR with subsequent symptom levels}

The mixed models analyses computing the association between SIR and psychiatric symptom levels at discharge, 3 months after discharge and 12 months after discharge are outlined in Table 3.

[Insert Table 3 about here]

A more positive SIR was a significant predictor of lower symptom levels at discharge $(p<0.01)$ and at 3 months after discharge $(p=0.02)$, and showed a trend towards 
predicting lower symptoms levels at 12 months after discharge, which however failed to reach statistical significance $(p=0.06)$.

Other significant predictors of symptom levels included age (significant positive association at 3 months after discharge), employment status (unemployment associated with increased psychiatric symptom levels at 3 months after discharge), baseline psychiatric symptom levels (significant positive association at all time points), and main psychiatric diagnosis (F2 associated with higher symptom levels at 3 months and 12 months after discharge). However, the predictive value of SIR was independent of the influence of these characteristics.

Association of SIR with subsequent social disability The mixed model analyses of the association between SIR and social disability at discharge, at 3 months and at 12 months discharge are outlined in Table 4.

[Insert table 4 about here]

A more positive SIR was significantly associated with lower social disability at 3 months $(p=0.01)$ and 12 months after discharge $(p=0.03)$.

Another significant predictor of social disability was the main psychiatric diagnosis (F2 associated with increased social disability at 12 months). Again, the predictive value of SIR was independent of this factor.

Interactions of SIR with treatment setting and main psychiatric diagnosis The interactions between SIR and treatment setting as well as diagnostic group were found to be non-significant in predicting symptom levels and social disability at all 
time points. Thus, there is no evidence that the association of SIR with outcomes varied between hospital and day hospital or across diagnostic groups.

\section{Comparison of research dropouts to completers}

Of the original 765 participants, 37 dropped out, i.e. did not complete any follow-up assessments. T-tests demonstrated that there was a significant difference between the SIR of research completers and research dropouts, with the mean SIR higher in completers. The effect size was small, with completer status explaining around $1 \%$ of the variance in CAT score $(t=-2.7$, d.f. $=764, p=0.007$, Partial $\eta 2=0.01)$.

\section{Discussion}

In a large multinational sample, patients' SIR to acute hospital and day hospital treatment was associated with outcomes over a one year period. A more positive SIR predicted lower symptom levels at discharge and three months later, and showed a trend towards doing so even 12 months after discharge. A more favourable SIR also predicted less social disability at 3 and 12 months after discharge. The associations were not explained by socio-demographic and clinical characteristics of the patients, which were controlled for as potential confounders. Also, there were no significant interaction effects with treatment setting or main psychiatric diagnosis in predicting outcomes. The study provides the most substantial evidence so far that SIR predicts outcomes in both hospital and day hospital settings and across all major psychiatric diagnostic groups.

Strengths of the study include the large sample size, which allowed us to control for confounding variables and test interaction effects in a multivariate analysis; a long follow up period with several assessments at different points of time; and reasonable follow-up rates. The use of observer rated rather than self rated outcomes shows that 
the association between SIR and outcomes can be explained neither by a consistent and general tendency of individuals for more positive or negative self ratings $(5,6$, 19), nor by a wish of patients to justify their initial views through providing corresponding outcome ratings. Furthermore, data was collected by researchers who were not involved in treatment, and data on SIR was collected on average six weeks before the first outcome assessment, thus minimizing the likelihood that a researcher's awareness of a patients' SIR could have influenced their outcome assessments. Finally, the study was conducted in five countries with the influence of the centre being controlled for in all analyses.

A limitation of this study is that the findings are based on patients who are willing to participate in a research trial. This may have introduced a selection bias and it is unclear whether the results are generalisable to those patients who cannot be recruited to trials and do not participate in research interviews. Moreover, we tested the association of SIR with outcomes without considering mediating factors such as the actual treatments patients received in the hospital and day hospital and during the follow-up periods.

The present findings represent a significant addition to the existing literature and provide much more substantive evidence on the predictive value of patients' SIR to complex interventions than had been hitherto available $(3,14,17,18)$. A new finding is that SIR predicts outcomes not only at discharge or after a short follow-up period, but also for a longer period of one year. This is consistent with a recent study on involuntary patients who assessed their views of treatment on the CAT within one week of admission, thus using the same assessment instrument, but somewhat later after admission than in this study. One year after admission, patients with more positive initial views of treatment had fewer involuntary re-admissions and were more likely to see the original involuntary admission as justified (20). One may conclude 
that patients' views of treatment at early stages are associated with outcomes beyond discharge.

In our study, the predictive value of SIR for symptom changes was not significant at one year, and thus seemed to diminish over time. Conversely, the predictive value of SIR for social disability was only significant at the two later follow-ups. This difference is likely to reflect the different nature of the two outcome criteria $(21,22)$. Whilst symptoms can fluctuate every day and be influenced short term, social disability is a more stable construct and usually requires more time for changes to materialise.

In the absence of evidence on the processes mediating SIR and outcomes, one can only speculate about why and how a more positive SIR is linked to more favourable outcomes across settings and diagnostic groups. There are at least four explanations which are not mutually exclusive: A) A positive SIR may indicate a higher motivation of patients to adhere to subsequent treatments so that the association is mediated by a better adherence to pharmacological and psycho-social treatments in the hospital or day hospital and during the follow-up period (10). B) Patients' SIR might reflect the quality of the therapeutic relationship between patients and treatment teams - as well as other non-specific factors - which can then directly or indirectly influence outcomes over time. C) The SIR may also reflect an internal preparedness of the patient for positive change in the given situation and thus a better responsiveness to psychiatric treatment. D) SIR may be linked to patient characteristics that have not been assessed in this and other studies, such as personality traits that may predict longer term outcomes after an acute crisis. 


\section{Conclusion}

Although the explanation for the association of SIR and outcomes is unknown, the finding that patients' outcomes up to a year later can be predicted simply by asking them within two days after admission whether they believe their treatment is right for them may be seen as astounding. It has clinical implications: patients' views on the appropriateness of treatment are important and should be listened to even within the first two days after admission, when clinicians might think that patients have not yet had sufficient experience of the new treatment to form a useful judgment. Although patients may still struggle to settle into the new hospital or day hospital setting and symptom levels can be high, patients' views of treatment are worth eliciting and listening to. SIR is easy to assess as part of routine clinical practice, and there is no reason to ignore such information that can be obtained at practically no cost. The association between SIR and outcome is not strong enough to make reliable predictions in individual cases. However, all other things being equal, clinicians may consider a positive SIR as a reassurance to continue with the current treatment plan, whilst a negative SIR may be a reason to consider changes to either the treatment setting or the specific treatment plan within it.

Three major challenges arise for future research. The first is to identify the processes mediating the association between SIR and outcomes. In particular, the role of the therapeutic relationship, treatment adherence, and specific treatment components should be studied. The second task is to explore factors that may influence patients' SIR, such as patients' expectations and health beliefs, the way in which the treatment plan is presented to them, or the quality of the initial therapeutic contact with the service. Such research should control for patient characteristics that have been found to be associated with their $\operatorname{SIR}(7,8)$. A third challenge is to develop interventions which aim to improve patients' SIR to treatment, either by moderating factors in 
patients' initial treatment experience or by changing patients' treatment plan to a more positively appraised one if they present a negative SIR. The next step will be to test to what extent SIR can be influenced and whether a SIR that is more positive as a result of such interventions is still associated with more positive outcomes. A pilot study which identified patients with negative SIR in a day hospital and then provided treatment in line with their wishes showed encouraging results (15), but much wider and more systematic experimental research is warranted.

\section{Acknowledgements}

Data collection for the EDEN study was facilitated by grants from the European Commission (Quality of Life and Management of Living Resources Programme: QLG4-CT-2000-01700), the Ronald-Ernst-Foundation for Health Services, Germany: (0302 Bach), the National Health Service Executive Organisation and Management Programme , United Kingdom, and the Ministry of Education, Slovakia (163/E394/SPUB-M/5 PR UE/DZ 30/2001-2002). Pfizer Pharmaceutical Co. supported travel and accommodation for EDEN project meetings.

All authors report no competing interests, and no financial or other relationships relevant to the subject of this article. The authors gratefully acknowledge the contribution of Duolao Wang to the statistical analyses reported in this publication. 


\section{References}

1. Awad AG, Hogan TP, Voruganti LN, Heslegrave RJ: Patients' subjective experiences on antipsychotic medications: implications for outcome and quality of life. Int Clin Psychopharmacol 1995; 10 Suppl 3: 123-132

2. Bartkó G, Herzog I, Békésy M: Predicting outcome of neuroleptic treatment on the basis of subjective response and early clinical improvement. J Clin Psychiatry 1987; 48: $363-365$

3. Bröker M, Röhricht F, Priebe S: Initial assessment of hospital treatment by patients with paranoid schizophrenia: a predictor of outcome. Psychiatr Res 1995; 58:77-81.

4. Eichler T, Schützwohl M, Priebe S, Wright D, Adamowski T, Rymaszewska J, Nawka P, Očvár L, Kitzlerova E, Rabboch J, Kallert T. Losses to follow-up in longitudinal psychiatric research. Epidemiol Psichiatr Soc 2008; 17: 138-147

5. Fakhoury W, Kaiser W, Röder-Wanner U, Priebe S: Subjective evaluation: is there more than one criterion? Schizophr Bull 2002; 28: 319-327.

6. Hansson L, Björkman T, Priebe S: Are important patient-rated outcomes in community mental health care explained by only one factor? Acta Psychiatr Scand 2007; 116: 113-118. 
7. Hansson L, Svensson T, Björkman T, Bullenkamp J, Lauber C, Martinez-Leal R, McCabe R, Rössler W, Salize H, Torres-Gonzales F, van den Brink R, Wiersma D, Priebe $S$ : What works for whom in a computer-mediated communication intervention in psychiatry? Moderators of outcome in a cluster randomised controlled trial. Acta Psychiatr Scand 2008; 118: 404-409.

8. Hofer A, Rettenbecher MA, Edlinger M, Kemmler G, Widschwenter CG, Fleischaker WW: Subjective response and attitudes towards antipsychotic drug therapy during the initial treatment period: a prospective follow-up study in patients with schizophrenia. Acta Psychiatr Scand 2007; 116: 354-366.

9. International Statistical Classification of Disease and Related Health Problems, Tenth Revision. World Health Organization, Geneva 2005.

10. Jonsdottor H, Friis S, Horne R, Pettersen KI, Andreassen OA: Beliefs about medications: measurement and relationship to adherence in patients with severe mental disorders. Acta Psychiatr Scand 2009: 119: 74-78.

11. Kallert T, Priebe S, McCabe R, Kienja A, Rymaszewska J, Nawka P, Očvár L, Raboch J, Stárková-Kaliŝová L, Koch R, Schützwohl M: Are day hospitals effective for acutely ill psychiatric patients? A European multicentre randomised controlled trial. J Clin Psychiatry 2007; 68: 278 - 287.

12. Kallert TW, Schützwohl M, Matthes C, EDEN-Study Group: The Client Sociodemographic and Clinical History Inventory. Dresden, Germany: Dresden University of Technology, Faculty of Medicine, 2000. 
13. Priebe S, Bröker M: Prediction of hospitalisations by schizophrenia patients' assessment of treatment: an expanded study. J Psychiatr Res1999; 33: 113-119.

14. Priebe S: Early subjective reactions predicting the outcome of hospital treatment in depressive patients. Acta Psychiatr Scand 1987; 76:134-138

15. Priebe S, Gruyters T: A pilot trial of treatment changes according to schizophrenic patients' wishes. J Nerv Ment Dis 1999; 187: 441

16. Priebe S, Gruyters T, Heinze M, Hoffmann C, Jäkel A: Subjective criteria for evaluation of psychiatric care: methods for assessment in research and routine care. Psychiatr Praxis 1995; 22:140-144.

17. Priebe S, Gruyters T: Patients' and caregivers' initial assessments of day hospital treatment and course of symptoms. Compr Psychiatry 1994; 35: 234-238.

18. Priebe S, Gruyters T: The importance of the first three days: predictors of treatment outcome in depressed inpatients. B J Clin Psychol 1995; 34: 229-236.

19. Priebe S, Kaiser W, Huxley P, Röder-Wanner U, Rudolf H: Do different subjective evaluation criteria reflect distinct constructs? J Nerv Ment Dis 1998; 186 : 385-392.

20. Priebe S, Katsakou C, Amos T, Leese M, Morriss R, Rose D, Wykes T, Yeeles K: Patients' views and readmissions one year after involuntary hospitalisation. B J Psychiatry $2009 ; 194: 49-54$ 
21. Priebe S: Social outcomes in schizophrenia. Br J Psychiatry 2007; 191 suppl. 50: s15-s20.

22. Priebe S, Watzke S, Hannsson L, Burns T: Objective social outcomes index (SIX): a method to summarise objective indicators of social outcomes in mental health care. Acta Psychiatr Scand 2008; 118: 57-63.

23. SAS Version 9.1. SAS Institute, Inc., Cary, NC, USA, 2005.

24. Schützwohl M, Jarosz-Nowak J, Briscoe J, Szajowski K, Kallert TW: Inter-rater reliability of the Brief Psychiatric Rating Scale (BPRS 4.0) and the Groningen Social Disabilities Schedule (GSDS-II) in a European multi-site randomised controlled trial on the effectiveness of psychiatric day hospitals. Int. J. Methods Psychiatr. Res. $2003 ; 12: 197-207$

25. Van Putten T, May R, Mander SR: Response to antipsychotic medication: The doctor's and the consumer's view. Am J Psychiatry 1984; 141: 16-19

26. Van Putten T, May R, Mander SR, Wittman LA: Subjective response to antipsychotic drugs. Arch Gen Psychiatry 1981; 38:187-190

27. Van Putten T, May R: Subjective response as a predictor of outcome in pharmacotherapy: The consumer has a point. Arch Gen Psychiatry 1978; 35:477-480

28. Ventura J, Lukoff D, Nuechterlein KH, Liberman RP, Green MF, Shaner A: Manual for the Expanded Brief Psychiatric Rating Scale. Int J Methods Psychiatr Res 1993; 3: $227-243$ 
29. Wiersma D, De Jong A, Kraaijkamp HJM, Ormel J: GSDS II: The Groningen

Social Disabilities Schedule, second version: manual, questionnaire, and rating form.

University of Groningen, Department of Social Psychiatry, Groningen 1990.

Table 1 Characteristics of the total sample $(n=765)$ and CAT (Clients Assessment of Treatment) score on day 3

\begin{tabular}{lll}
\hline & N or mean & \% or SD \\
Characteristics & & \\
\hline Centre, N (\%) & 170 & 22 \\
Czech Republic & 131 & 17 \\
Germany & 217 & 28 \\
Poland & 159 & 21 \\
Slovakia & 88 & 12 \\
United Kingdom & & \\
Treatment setting, N (\%) & 374 & 49 \\
Inpatient care & 391 & 51 \\
Day hospital care & 66 & 62 \\
Length of stay (days), mean (SD) & 481 & 63 \\
Gender, female, n (\%) & 40 & 12 \\
Age (years), mean (SD) & 121 & 16 \\
Living alone, N (\%) & 12 & 3 \\
Education (years), mean (SD) & 230 & 30 \\
Employed, N (\%) & & \\
ICD-10 diagnosis, N (\%) & 218 & 29 \\
Diagnosis F20-F29 & 333 & 43 \\
Diagnosis F30-F39 & 214 & 28 \\
Diagnosis F40-F59 & 7.6 & 1.9 \\
CAT score on day 3, mean (SD) & &
\end{tabular}


Table 2 Mean BPRS (Brief Psychiatric Rating Scale) and GSDS (Groningen Social Disabilities Schedule, Second Revision) scores at baseline, discharge, 3 months and 12 months post-discharge

\begin{tabular}{lllll}
\hline Measure & $\begin{array}{l}\text { At } \\
\text { Baseline }\end{array}$ & $\begin{array}{l}\text { At } \\
\text { Discharge }\end{array}$ & $\begin{array}{l}\text { At 3 } \\
\text { months }\end{array}$ & $\begin{array}{l}\text { At 12 } \\
\text { months }\end{array}$ \\
\hline BPRS & & & & \\
N & 765 & 686 & 606 & 541 \\
Mean & 46.7 & $34.9^{\mathrm{a}}$ & $37.2^{\mathrm{ab}}$ & $36.2^{\mathrm{abc}}$ \\
SD & 9.9 & 7.5 & 9.8 & 9.6 \\
\hline GSDS & & & & \\
N & 727 & 213 & 325 & 308 \\
Mean & 9.37 & $7.17^{\mathrm{a}}$ & $6.27^{\mathrm{ab}}$ & $6.03^{\mathrm{ab}}$ \\
SD & 0.54 & 3.93 & 4.56 & 4.74 \\
\hline
\end{tabular}

Results of paired t-tests:

a Significant difference from baseline $\quad(p<0.01)$

b Significant difference from discharge $(p<0.01)$

c Significant difference from 3 months $(p<0.01)$ 
Table 3 Main effects of subjective initial response and patient characteristics on BPRS score at discharge, month 3 and month 12 in mixed model analyses controlling for baseline symptom level, treatment setting and length of stay in hospital or day hospital

$$
\text { Discharge }
$$

$$
\text { Month } 3
$$

\begin{tabular}{|c|c|c|c|c|c|c|c|c|c|c|c|c|}
\hline Variables & Coefficient & \multicolumn{2}{|c|}{$95 \% \mathrm{Cl}$} & $\mathrm{P}$ & Coefficient & \multicolumn{2}{|c|}{$95 \% \mathrm{Cl}$} & $\mathrm{P}$ & Coefficient & \multicolumn{2}{|c|}{$95 \% \mathrm{Cl}$} & $\mathrm{P}$ \\
\hline Gender- female vs male & -0.030 & -0.076 & 0.016 & 0.196 & -0.043 & -0.107 & 0.020 & 0.178 & 0.016 & -0.050 & 0.081 & 0.641 \\
\hline Age (years) & 0.002 & 0.000 & 0.004 & 0.063 & 0.003 & 0.000 & 0.005 & 0.040 & 0.001 & -0.002 & 0.004 & 0.436 \\
\hline Education (years) & -0.004 & -0.012 & 0.004 & 0.376 & -0.001 & -0.012 & 0.010 & 0.805 & 0.000 & -0.012 & 0.011 & 0.962 \\
\hline Living status - not alone vs alone & 0.046 & -0.017 & 0.108 & 0.151 & 0.019 & -0.064 & 0.102 & 0.655 & 0.023 & -0.065 & 0.111 & 0.611 \\
\hline $\begin{array}{l}\text { Employment status- employed vs unemployed } \\
\text { ICD-10 Diagnosis }\end{array}$ & 0.038 & -0.011 & 0.087 & 0.124 & 0.067 & 0.000 & 0.133 & 0.049 & 0.066 & -0.003 & 0.136 & 0.061 \\
\hline F3 vs F2 & -0.042 & -0.098 & 0.015 & 0.15 & -0.141 & -0.219 & -0.060 & $<0.001$ & -0.135 & -0.216 & -0.054 & 0.001 \\
\hline F4 vs F2 & 0.006 & -0.057 & 0.068 & 0.862 & -0.011 & -0.097 & 0.075 & 0.798 & -0.076 & -0.167 & 0.014 & 0.098 \\
\hline Subjective Initial Response (CAT score on day 3) & -0.019 & -0.031 & -0.007 & 0.033 & -0.021 & -0.038 & -0.004 & 0.016 & -0.016 & -0.034 & 0.001 & 0.064 \\
\hline
\end{tabular}

Month 12 
Table 4 Main effects of subjective initial response and patient characteristics on GSDS score at discharge, month 3 and month 12 in mixed model analyses controlling for baseline social disability level, treatment setting and length of stay in hospital or day hospital

Discharge Month 3 Month 12

\begin{tabular}{|c|c|c|c|c|c|c|c|c|c|c|c|c|}
\hline Variables & Coefficient & $95 \%$ & & $\mathrm{P}$ & Coefficient & 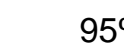 & $\mathrm{Cl}$ & $P$ & Coefficient & & $\mathrm{Cl}$ & $\mathrm{P}$ \\
\hline Gender- female vs male & -0.065 & -0.152 & 0.023 & 0.149 & -0.041 & -0.131 & 0.049 & 0.373 & 0.044 & -0.053 & 0.142 & 0.375 \\
\hline Age (years) & 0.001 & -0.002 & 0.005 & 0.461 & 0.002 & -0.002 & 0.006 & 0.344 & 0.001 & -0.003 & 0.005 & 0.580 \\
\hline Education (years) & 0.012 & -0.146 & 0.171 & 0.375 & -0.091 & -0.244 & 0.062 & 0.243 & -0.059 & -0.222 & 0.104 & 0.478 \\
\hline Living status - not alone vs alone & 0.063 & -0.053 & 0.179 & 0.287 & -0.029 & -0.151 & 0.092 & 0.638 & -0.068 & -0.202 & 0.067 & 0.322 \\
\hline $\begin{array}{l}\text { Employment status- employed vs unemployed } \\
\text { ICD-10 Diagnosis }\end{array}$ & 0.046 & -0.049 & 0.142 & 0.338 & 0.089 & -0.005 & 0.184 & 0.063 & & & & \\
\hline F3 vs F2 & -0.050 & -0.156 & 0.055 & 0.350 & -0.073 & -0.184 & 0.037 & 0.194 & -0.184 & -0.305 & -0.062 & 0.003 \\
\hline $\mathrm{F} 4$ vs F2 & -0.038 & -0.158 & 0.082 & 0.535 & -0.060 & -0.180 & 0.060 & 0.324 & -0.120 & -0.253 & 0.012 & 0.075 \\
\hline Subjective Initial Response (CAT score on day 3 ) & -0.020 & -0.044 & 0.004 & 0.108 & -0.031 & -0.056 & -0.006 & 0.014 & -0.029 & -0.056 & -0.003 & 0.031 \\
\hline
\end{tabular}


Lars Hansson

Lars.Hansson@med.lu.se

Division of Nursing, Room nr: HSC 286E, Hs 65, Lund University, Sweden.

Tel +46 046-2221842

Fax 46 046-2221824

Christoph Lauber

Christoph.Lauber@liverpool.ac.uk

School of Population, Community and Behavioural Sciences, University of Liverpool

Tel +44 (0)151 7064140

Thomas Becker

psychiatrie@bkh-guenzburg.de

Department of Psychiatry and Psychotherapy, Ulm University, Sweden

Phone +49-8221/96-2002

Fax (+49) 8221 / 96-2400 